\title{
Application of Research Priorities by Funding Agencies in Europe
}

\author{
P.E. Fricker, Bern \\ (Secretary-General of the Swiss National Science Foundation and Past Chairman of the Committee of the European Science \\ Research Councils)
}

It is extremely difficult to establish a coherent national research policy just because the sources of financing and the interest and responsibility of the parties involved are all so varied. In Switzerland, for example, about $75 \%$ of the costs for $R \& D$ are carried by private enterprise, while about $25 \%$ are paid through the Federal and Cantonal governments. Under these conditions it is understandable that direct governmental influence on industrial $R \& D$ is limited. A comparable situation exists in other countries too, although the government contributions generally represent a bigger share.

One has also to consider the complexity and the often unpredictable development of the many scientific disciplines that have to be taken into account, as well as the necessity for maintaining academic freedom. It is hardly surprising therefore, that the United States and the countries in western Europe, at least, have not pursued over an extended period of time a science policy that is well-defined. The question arises whether it is possible or even sensible to formulate such an encompassing policy which could inhibit the flexibility that is indispensable for scientific development.

On the other hand, as many national science budgets are stagnating or declining, the pressure for a more explicit policy is mounting and is reinforced by the steady rise in research costs. This is evident in the case of research activities which are directly or indirectly - supported by government funds. Research councils and analogous bodies are increasingly expected to play an active part in policy-making, especially by setting research priorities.

Against this background of change, an attempt is made to outline the methods used to introduce and apply research priorities in western Europe with emphasis on funding agencies which are primarily concerned with the promotion of basic research. A study of the European Science Research Councils, a Standing Committee of the European Science Foundation, published under the title ESRC Review No. 12 , "Science Research Council Priorities in Europe", Stockholm 1977*, and other information from funding bodies have been taken into consideration in the following survey.

*Available from the ESF Strasbourg
It is difficult to circumscribe the meaning of the term "research priority" which can refer to different levels (for instance regional, national or international) and time periods (short- or long-term).

In the present context, a research priority is defined as a preference for a specific scientific field, subject or type of support, for example: solid state physics (scientific field), programme on investigation of acidic rain effects (subject), determination of the number of fellowships (type of support). The preference, which necessitates a process of selection, is usually expressed in terms of special financial assistance.

\section{Basic Policy}

The approach of the funding agencies in western Europe towards science policy matters, while consistent with their concrete tasks, is generally pragmatic and their rôle in establishing priorities depends on their structure and method of operation. A first distinction can be made between funding bodies which maintain their own laboratories or institutes (among them the Centre National de la Recherche Scientifique in France, the Consiglio Nazionale delle Ricerche in Italy, the National Environmental Research Council in Great Britain and, to a lesser extent, the Deutsche Forschungsgemeinschaft) and organisations which do not have their own facilities (for instance the Swiss National Science Foundation, the Scientific Research Support Foundation of Austria, the Norwegian Research Council for Science and the Humanities). There are other differences, notably in the processes of decision-making and the interrelationship with government: indeed each council has its own personality, a factor which has to be taken into account when councils collaborate internationally. Nevertheless, when it comes to the methods of research support, councils have adopted similar solutions, contributing through, for example, research project grants (for projects of qualified researchers), fellowship programmes (to advance young researchers) and publication grants (to facilitate scientific publications).

It is evident that funding agencies maintaining their own institutes can more easily apply policy guide-lines and introduce priorities because they have a direct influence on the infrastructure. The other councils, which are primarily concerned with unsolicited proposals (applications submitted on the initiative of researchers), are more limited in their actions as they have to rely mainly on the existing infrastructure of the universities and other research institutes.

Establishing Priorities Inevitably Implies Establishing Posteriorities

Research priorities become established mainly as a result of judgments made by the scientific community which the research councils are serving. Influence on the selection procedure is also exercised by government. A comparison between different countries indicates that the main criteria for selection are rather similar: scientific aspects predominate over social considerations. New concepts or techniques, which can have a decisive impact on the scientific advance in a given field, exert a strong influence.

Although the support of individual research projects is not discussed here, it should be mentioned that the evaluation for funding of research proposals, mostly through peer review, represents a priority selection. At this level, the view is still often expressed that scientific excellence is the only true priority and this must not be taken lightly. Defining priorities should not lead to the support of mediocre research work; a high scientific standard has to be maintained. Even when priorities have been laid down, several factors have to be examined before they can be applied, including the scientific feasibility, the extent of the research potential and the estimated costs.

For priorities to be implemented, financial support has to be concentrated in the designated area and, as the total budget usually does not increase, this necessarily means that economies have to be made elsewhere. This in turn signifies that low priority areas, sometimes referred to as "posteriorities", have to be defined. The determination of posteriorities, i.e. areas of diminishing support, is a most difficult and ingrate exercise which research councils and advisory bodies try to avoid. Despite this, a few funding agencies, including the Swiss National Science Foundation for its four year plan 1984/87, have explicitly designated such low-priority subjects within main disciplines like astronomy, chemistry, physics and environmental sciences.

It takes years to build up a qualified research potential in a given scientific field, 
yet a lack of support can rapidly destroy this potential. In order to maintain the necessary continuity of the principal research efforts, priorities and especially posteriorities should be applied gradually, even though fast action may sometimes be required for priorities having only a short life-span.

Research councils dispose of several means for putting research priorities into practice. Depending on specific needs, the following principal instruments are employed: co-ordinated cooperation, national research programmes, international cooperation and fellowship programmes.

\section{Priority Programmes}

A study of several different priority programmes indicates that, in general, three main phases can be distinguished: preparation, decision and execution. A fourth step may consist of the evaluation and application of the results. The key question as to who finally decides on a priority programme cannot be clearly answered. Depending on the nature of the programme, the research councils or the government are competent. Not surprisingly their motives are not same. The councils are driven by scientific motives; for the government, political motives are also important. Governmental influence is usually strongest during the preparation of a programme and partly also in connection with the final decision. The other phases, especially the executive stage, are dominated by the research councils.

For most priority programmes, the time elapsing between the initiative and the final decision ranges from six months to over two years. It is essential that ways are found of reducing this relatively long timespan during the preparative phase.

A delicate matter concerns the utilization and implementation of the results obtained from priority programmes. Experience shows that the scientific conclusions do not always coincide with the views and ideas of government or parliament. Under these circumstances, mutual confidence and tolerance are indispensable.

\section{Conclusions}

In summary, the research councils and analogous bodies in western Europe are increasingly required to apply priorities in their support of research and to see that well-founded scientific contributions are made to the solution of national problems. They are the guardians of public funds and their activity has to be placed in the framework of a general national policy. Within these boundaries, however, a certain degree of autonomy and flexibility must be secured. Only then can the research councils fulfil the principal task entrusted to them, namely the support of creative basic research which is the cornerstone of industrial development and also of a nation's welfare.

\title{
Laser Induced Chemical Vapour Deposition
}

\author{
Dieter Bäuerle, Linz
}

(Angewandte Physik, Johannes Kepler University)

Laser-induced chemical vapour deposition (LCVD) is a new technique for single step local deposition or direct writing of thin films of metals, semiconductors or insulators. The lateral dimensions of these films can be varied from about $0.5 \mu \mathrm{m}$ up to several $\mathrm{mm}$. Holographic methods may permit single-step deposition of complete material patterns. In pyrolytic LCVD, the deposition rates are so high that three dimensional structures can be produced.

Flat structures with widths down to micron-size are needed in many areas of technology, such as microelectronics or integrated optics. Such structures are presently produced by standard chemical vapour deposition (CVD) together with sequential application of mechanical masking or photolithographic methods. These standard techniques, which require several production steps, are well established for large area planar substrates. On the other hand, these techniques are not very suitable for deposition of structures on nonplanar substrates. Furthermore, the increasing complexity and miniaturization of systems require controlled area coating techniques which avoid thermal or chemical cycling of entire partially fabricated devices. In such cases, LCVD is an extremely promising technique.

Applications of complex three dimensional structures produced by pyrolytic LCVD can only be speculated on. In this new and fascinating field only the first step has been taken: recently, rods of single crystalline Si have been grown within the beam of a laser - without any crucible and in an otherwise cold atmosphere. The possibility of producing non-equilibrium materials or materials which form only under extreme conditions, e.g. at high temperatures together with high pressures, is obvious.

In standard CVD, the substrate is immersed in an atmosphere of gaseous reactants in a closed reactor. The substrate is uniformly heated; the chemical reaction which leads to material deposition is thermally activated near or at the hot surface of the substrate. As a consequence, one obtains deposition of extended films.

LCVD can be based on reactions which are initiated mainly pyrolytically or photolytically, or on a combination of both.

Pyrolytic LCVD is based on local substrate heating by laser light which is not absorbed by the gaseous molecular species.
Apart from nucleation (see below), the microscopic mechanism for decomposition is the same as in conventional CVD. Therefore, the wide variety of deposition reactions used in conventional CVD can be taken over to LCVD. However, in LCVD, the chemical reaction is confined to the hot spot which is produced on the substrate or on the material deposited already - by the absorbed laser light.

The situation is quite different for photolytic LCVD. In this case the laser radiation breaks chemical bonds directly, i.e. non-thermally. Since most molecular bond energies are several $\mathrm{eV}$, ultraviolet (UV) laser light is generally required. Besides dissociative electronic excitation, dissociation of molecular species can also be achieved by multiphoton vibrational excitation with infrared laser light of suitable frequency.

\section{Surface Nucleation}

Nucleation based on purely thermal processes has been extensively studied in connection with standard thin film growth techniques. In photolytic LCVD, however, non-thermal dissociation of reactant molecules which are adsorbed at the surface of the substrate plays a basic role. This has been investigated mainly in the deposition of metal films, especially from metal-alkyls, such as $\mathrm{Cd}\left(\mathrm{CH}_{3}\right)_{2}$ and $\mathrm{Al}_{2}\left(\mathrm{CH}_{3}\right)_{6}$.

Irradiation with UV laser light of suitable frequency results in photodissociation of the adsorbed molecules, producing free metal atoms or clusters of atoms which act as nucleation centres for atoms produced in the gas phase. Moreover, there is strong evidence that, even in cases where film growth proceeds by thermal dissociation of the molecules, nucleation may be initiated by single or multiphoton dissociation of adsorbed molecules. Evidence for such processes has been obtained during $\mathrm{Cd}$ and $\mathrm{Ni}$ deposition from $\mathrm{Cd}\left(\mathrm{CH}_{3}\right)_{2}$ and $\mathrm{Ni}(\mathrm{CO})_{4}$ on transparent substrates with visible $\mathrm{Kr}^{+}$and $\mathrm{Ar}^{+}$laser radiation.

\section{Pyrolytic Deposition}

The fundamental parameter in pyrolytic deposition is the local temperature within the reaction zone. The temperature rise on a surface due to absorption of laser radiation is a function of the reflectivity and the thermal conductivity of the solid. While at the very beginning of growth these quantities are determined solely by the sub- 